\title{
Gravitational Waves
}

\author{
B. F. Schut $z^{i 2}$
}

${ }^{a}$ Department of Physics and Astronomy, University of Wales College of Cardiff, P.O. Box 913, Cardiff CF2 3YB, United Kingdom

In the last few years there have been a number of significant developments in research towards the detection of gravitational radiation from astronomical objects. The construction of 3 largescale $(3-$ or $4-\mathrm{km})$ interferometric detectors has been funded; new high-sensitivity bars are under construction; there is a serious proposal to ESA to place an interferometric detector in space; the data from the first coincidence observation using two interferometers has been used to put upper limits on gravitational wave bursts; and new theoretical studies of the relativistic two-body problem have shown that observations of the coalescences of two neutron stars or black holes from binary orbits can provide detailed information about the stars' masses and spins - but only if we can solve the two-body problem better than we have done so far. These developments are reviewed here.

\section{INTRODUCTION}

Since my last talk at a TAUP meeting, in 1989 [1], there has been remarkable progress toward the goal of detecting gravitational waves from astronomical events. Both on the experimental and theoretical fronts, recent developments have given us a much clearer idea of what we can expect to learn, and on what timescale. Rather than review the entire field, I will simply update here what I think have been the most significant advances.

The first main section will be an update on detector developments. Large-scale interferometer projects to build two $4-\mathrm{km}$ detectors in the United States (the LIGO project [2]) and to build a similar $3-\mathrm{km}$ instrument in Italy (the VIRGO project [3]) have received funding and seem certain to go ahead. The LIGO team estimate they will begin observing in 1999, and they should steadily improve their sensitivity after that. The first ultracryogenic bar detector has been cooled to below $100 \mathrm{mK}$ at Frascati, and a similar bar at Stanford is under development. With a little luck, such bars could make a detection before 1999 . Meanwhile, ESA is studying a proposal (called LISAG at the moment) to place an interferometer in space to search for low-frequency radiation from giant black holes and ordinary binary star systems.

In the next section, I will give the first re- sults from the analysis of data from the first coincidence experiment between two interferometers. The data set has revealed no surprises in terms of gravitational wave events, but it has provided considerable reassurance that it will not be hard to get the large detectors to operate for long periods of time at near-optimal sensitivity.

In the final main section I discuss a theoretical development: studies of binary systems in general relativity have revealed that the new design goals of LIGO and VIRGO could in fact reveal rich detail about the component members of binaries that are observed to coalesce. But this comes at a price: we have to be able to solve and understand the 2-body problem much more accurately than we do at present.

It may help at this point to fix notation by reminding readers of a few elementary facts about gravitational waves. Travelling at the speed of light, they carry the changes in the gravitational field caused by moving masses. They are transverse waves that act through gravitational tidal forces, which means that their effect on a detector (say a stretching of its length by an amount $\delta \ell$ ) is proportional to its size $\ell$. Thus, the wave can be characterized by its amplitude $h=\delta \ell / \ell$, which falls off with increasing distance $r$ from the source as $1 / r$. Typical amplitudes are $10^{-18}$ for strong waves from our own Galaxy, and $10^{-21}$ or smaller for waves from nearby clusters of galaxies, like the 
Virgo cluster. Detectors sense the tidal action of the waves, either by directly detecting changes in the proper distance between free masses (interferometers do this) or by detecting the ring-down of a resonant mass that has been stretched by an impulsive wave (bar detectors).

Gravitational waves can come from many sources. Stellar sources like supernova explosions or the coalescences of compact-object binaries can emit strong radiation with frequencies up to $1 \mathrm{kHz}$ or more. Pulsars can emit radiation at twice their rotational frequency. There may be a cosmological background of radiation at nearly all frequencies. Giant black holes in galactic centres can emit radiation at milli-Hertz frequencies when perturbed by infalling neutron stars or when merging with other black holes.

\section{DETECTOR DEVELOPMENTS}

\subsection{Ultracryogenic bar detectors}

The oldest type of detector is the bar detector, which was first developed by J. Weber at the University of Maryland. For a full review of detector methods, see the comprehensive book edited by Blair [4]. Bar detectors sense the excitation of the normal modes of the bar by a gravitational wave. For this reason, they are usually narrow-band detectors at frequencies near $1 \mathrm{kHz}$. To reduce the thermal noise background, modern bars are cooled to cryogenic temperatures. The coolest bar so far constructed is the Nautilus bar of the Rome group, in their laboratory at Frascati. They managed to cool it below $100 \mathrm{mK}$ last year [5], and are at present cooling it again with its full suite of instrumentation. The goal is to reach below $70 \mathrm{mK}$, at which point it might be possible to reach sensitivities well below $h=10^{-19}$, even approaching $10^{-20}$.

The group at Stanford University are also constructing such a bar, and the Rome group have plans for at least one further bar in Italy. With a network of 3 such detectors, it is possible that gravitational waves from a gravitational collapse event anywhere in our local group of galaxies could be detected. This might happen once in ten years or so, giving such a network a good chance of making the first detection before 1999, when they will probably be overtaken in sensitivity by the interferometers described next.

\subsection{Interferometric detectors}

The LIGO and VIRGO project have grown out of the prototype interferometers that have been operated for some years by Glasgow University, by the Max Planck Institute for Quantum Optics, and by the California Institute of Technology. They are broadband detectors, with the frequency range limited in a fundamental way at low frequencies by environmental disturbances and at high frequencies by the available amount of laser power.

Present prototypes have sensitivities of about $h=10^{-18}$ over a bandwidth from about $500 \mathrm{~Hz}$ to 2 or $3 \mathrm{kHz}$. The LIGO and VIRGO projects as now approved envision reaching a first-stage sensitivity nearly to $10^{-21}$ over a bandwidth from perhaps $40 \mathrm{~Hz}$ or lower, up to $1 \mathrm{kHz}$. This could happen as early as 1999. But once built, they can in principle be improved to something like $10^{-22}$ over a range that extends down to 20 or even $10 \mathrm{~Hz}$. This might take another three to five years.

The LIGO project intends to build its detectors in Hanford, Washington, and in Livingston Parish, Louisiana. The project is a collaboration between the California Institute of Technology and the Massachusetts Institute of Technology. Its headquarters are at Caltech. Construction of the detector in Washington should begin early in 1994. At a cost of $\$ 100 \mathrm{M}$ each, the detectors are designed to allow extensive scientific development over a number of years. Essentially all the cost goes into the vacuum system and buildings. Once constructed, the further development of the optics and other systems to improve sensitivity will be relatively inexpensive. Considerable effort is being put into the design of the control systems that will allow the detectors to respond automatically to problems, such as the loss of an interferometric fringe, or a chance large environmental disturbance. In this way, these detectors are expected to be able to observe for months at a time with very few interruptions and with reasonably constant sensitivity.

The VIRGO project is a wide-ranging collaboration among many groups in France and Italy, directed from Pisa and Orsay. It has only recently been funded by the Italian and French authori- 
ties (INFN and CNRS, respectively). Its $3-\mathrm{km}$ detector will probably be built just outside Pisa, but other sites are under investigation. The design goals are substantially the same as for LIGO, although VIRGO places more emphasis on lowfrequency operation and the search for pulsars. Again, the system will be upgradeable, and great emphasis is being placed on automatic control. VIRGO may begin operation in 1999 or 2000 .

Having three such detectors is not a luxury: it is the minimum one needs for extracting the full information from the waves. This is because detectors are not directional: their quadrupolar antenna patterns offer little information about the arrival direction of the waves. The incident direction can only be inferred by triangulating on the different arrival times of the waves at three different detectors. [6]

It follows that the VIRGO and LIGO teams need to cooperate on exchanging their data in order to gain maximum information from it. Discussions are already underway to ensure compatibility of data formats and so on.

Regardless of whether gravitational waves are detected first by bar detectors, real astrophysical information is only likely to be extracted from gravitational waves by the interferometers. Their wide bandwidth and improved sensitivity makes them true gravitational wave observatories. I shall give an example of the sort of information obtainable in Section (4) on binary coalescence below.

\subsection{An interferometer in space}

The dominant noise that a ground-based interferometer will sense at low frequencies is gravity-gradient noise from masses in motion on the Earth. These masses can be passing trucks or large air masses in high-pressure systems. They act directly through their gravitational fields, so they cannot be screened out. The only way to escape them is to go into space.

Recently two proposals $[7,8]$ have been submitted to the European Space Agency in response to its call for proposals for its M3 mission, a medium-cost launch expected around 2004. One proposal envisions an Earth-orbiting array of 6 spacecraft with laser transponders, making 3 arms the other a solar-orbiting array of 4 . The response of ESA is encouraging: the proposals have been merged into a single one that is one of 6 proposals selected for further study. In one year the study will report on the best approach, and the 6 will be winnowed to 4 . The study goes by the rather awkward acronym of LISAG.

The most spectacular source of gravitational waves in the milliHertz region of the spectrum accessible from space is the coalescence of two massive black holes in the centre of a distant galaxy. Such events presumably occur occasionally, because it is now commonly believed that such black holes are present in many - if not most -- galaxies, and because we also see galaxies merging. Gravitational waves from such an event would have a unique signature and would stand out with a signal-to-noise ratio of several thousand even for the most distant galaxies we can see.

Other possible sources include neutron stars falling into massive black holes, which again must occur from time to time, but which would be harder to pick out. In our Galaxy, radiation would be detectable from binary systems composed of white dwarfs, neutron stars, or black holes, and from some known close binary systems. Figure 1 shows the sensitivity curve that was given in the proposal for a solar-orbiting detector, called LISA. It is illustrative of what might be achieved by a space-based detector with current technology, but the study now going on should render these predictions more precise.

\section{INTERFEROMETER COINCIDENCE EXPERIMENT}

In order to demonstrate the practicality of making long-term coincident observations with interferometers and of analyzing the data, an observation lasting 100 hours was undertaken in 1989 using the prototype detectors of the Max-PlanckInstitute for Quantum Optics and of Glasgow University. This was the first time that two interferometers have been run in coincidence for any length of time. The broadband sensitivity of both detectors at the time of the experiment was a litthe above $h=10^{-17}$, which is poorer by a factor of about 10 than prototypes could achieve today. This sensitivity might have been sufficient. to detect a nearby $(1 \mathrm{kpc})$ gravitational collapse event in our Galaxy, the probability of which in 


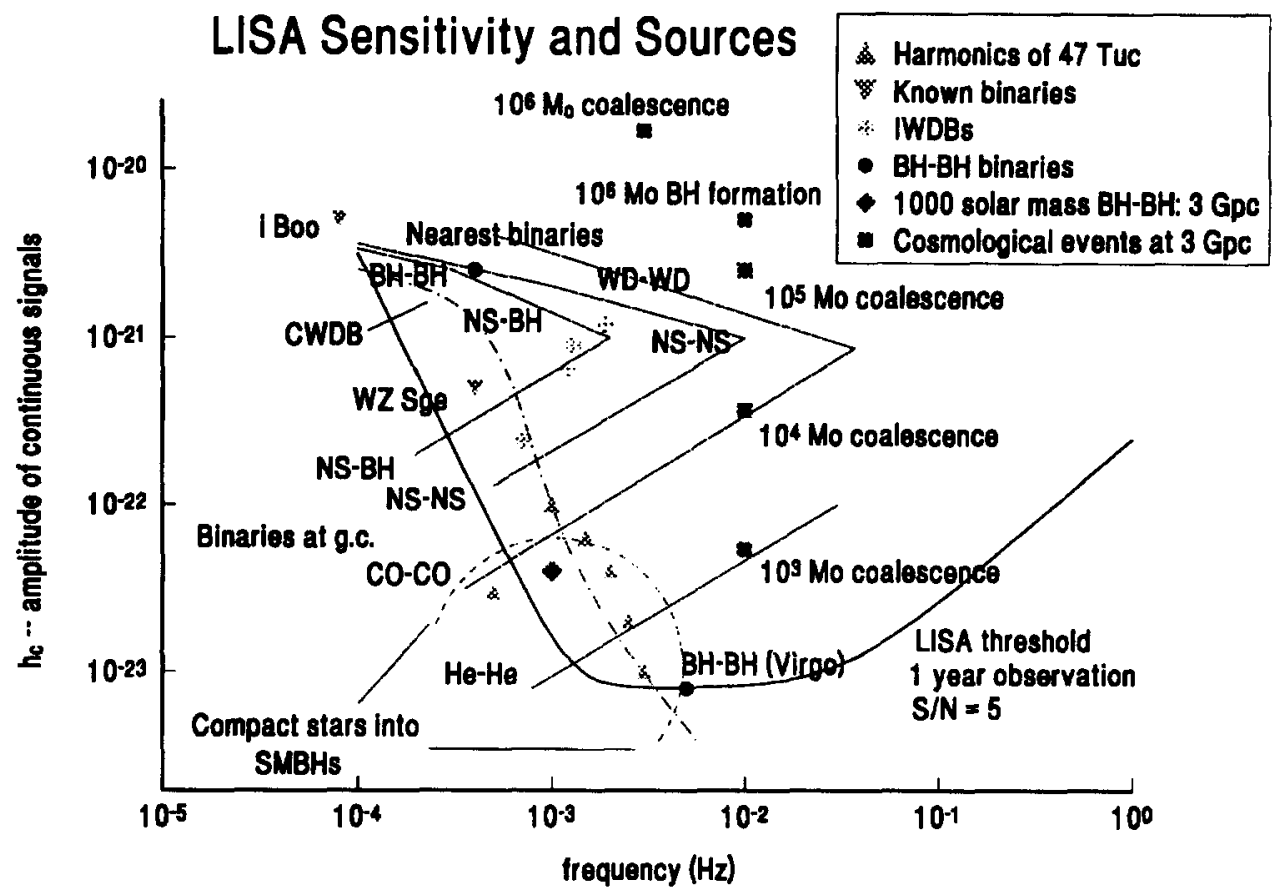

Figure 1: Strength of various sources and the sensitivity curve of LISA. The sensitivity curve is for an integration time of 1 year, a signal-to-noise ratio $S / N$ of 5 , and an isotropic average over source directions. The curve labeled "CWDBs" is an estimate of the confusion limit due to both galactic and extragalactic close white dwarf binaries.

The characteristic strain amplitude $h_{c}$ is shown for different types of periodic and quasiperiodic sources. The signals for some known binaries are given. The signal one would get from binaries of different types and frequencies at the Galactic centre is shown by rising lines. The falling lines show the signal from the nearest expected compact-object binary, if the formation rates assumed in the text are correct. These two sets of lines join at the highest-frequency binary one could expect. The expected shortest period black-hole-black-hole binary in the Virgo cluster is also shown.

The dashed arc encloses a region where one might find relativistic binaries consisting of a compact star in an eccentric orbit around a black hole of mass of order $10^{6} M_{\odot}$. Cosmological coalescing binaries of various masses and the formation of a supermassive black hole are shown at $3 \mathrm{Gpc}$. Because their signals do not last as long as a year, the amplitude shown is the equivalent amplitude of a continuous source that would have the same $S / N$. The coalescence points are given at arbitrary frequencies, since the signal "chirps" with time. 
any 100-hour period may be between $10^{-5}$ and $10^{-6}$. In fact, the experiment did not find any evidence for such an event, setting an upper limit of $3 \times 10^{-16}$ on the amplitude of any gravitational wave passing through the detectors during an effective observing period of about 62 hours. These results are being published separately [9].

\subsection{Description of the experiment}

Curve a of Figure 2 displays the strain sensitivity of the Glasgow interferometer during a typical period of the experiment. Above about $800 \mathrm{~Hz}$ the instrument operated close to its shotnoise (laser-power) limited sensitivity. The excess noise below $800 \mathrm{~Hz}$ is caused mainly by ground vibrations and mechanical resonances.

The important number for detection of supernova bursts is the broadband sensitivity in a bandwidth from $800-1250 \mathrm{~Hz}$. A rough estimate of about $10^{-17}$ for the rms strain noise level in the Glasgow detector in this bandwidth can be inferred from the curve. The Garching detector's noise curve (labelled b) displays features that are similar to those seen in the Glasgow data, and it furthermore shows the effects of an antialiasing filter $(4 \mathrm{kHz})$ and a low-frequency cut-off at $320 \mathrm{~Hz}$. (Filters were also used on the Glasgow data but have been removed from Figure 2 a by using calibration information.) The rms strain noise of the Garching detector in the range from 800 to $1250 \mathrm{~Hz}$ was also around $10^{-17}$ near the start of the observing period but later deteriorated by about a factor of two to three.

The Glasgow detector produced $20 \mathrm{~GB}$ of data, and the Garching detector about $15 \mathrm{~GB}$, during the experiment. These large data sets are probably smaller than will be produced by LIGO and VIRGO during comparable amounts of time.

Interferometers are intrinsically more difficult to operate than bar detectors, since they involve a number of active control systems that must constantly be monitored and occasionally corrected. As remarked above, the designs for the large-scale interferometers incorporate many control features designed to minimise the need for operators to intervene with the running of the detectors. The prototypes do not incorporate such features, since they were designed as scientific development testbeds rather than as observatories. It was therefore encouraging that the prototypes performed so well: they acquired data simultaneously during $88 \%$ of the experiment, and operated close to their optimum sensitivity simultaneously for $62 \%$ of the experiment.

\subsection{Analysis of the data}

Many of the general issues that arise in the analysis of gravitational wave data have been addressed in recent monographs $[10,11]$. A preliminary analysis of the Glasgow data has already appeared [12], and the Garching data set has been searched for a pulsar signal [13]. Our concern in Cardiff was to develop an analysis method that could serve as a prototype of the systems that will have to search the data from the large-scale detectors and keep up with the continually arriving data. The program needed to search for shorttime-scale bursts that would be produced by supernova explosions, for coalescing binaries, and for other events. The program has been described fully elsewhere [14]. In this section I will only describe our results for supernova-type bursts. A schematic of the analysis pipeline is shown in Figure 3.

Our analysis method was to compute the overlap integral between the data stream and a filter weighted with the power spectral density of the noise in the data stream to suppress regions of frequency where the data is very noisy. This is the well-known matched-filtering method. We adopted a simple model of a supernova signal, essentially just a fiat spectrum up to $1250 \mathrm{~Hz}$.

The programs produced lists of events from each data stream, which are essentially times when the filtered data cross a certain threshold at a time when the detectors were working acceptably. A coincidence is defined as a pair of events from the two data streams that occur within the acceptance window given by the gravitational-wave travel time. The list of coincidences inevitably contains some with very large amplitude, and one has to assess their significance. It is important to formulate a priori criteria for accepting events, without reference to the specific properties of the data set. Our analysis proceeded through 3 levels of vetoes.

At the first level, we looked at housekeeping data and applied vetoes if the detectors were not performing correctly or if an environmental disturbance had occurred which may have affected 


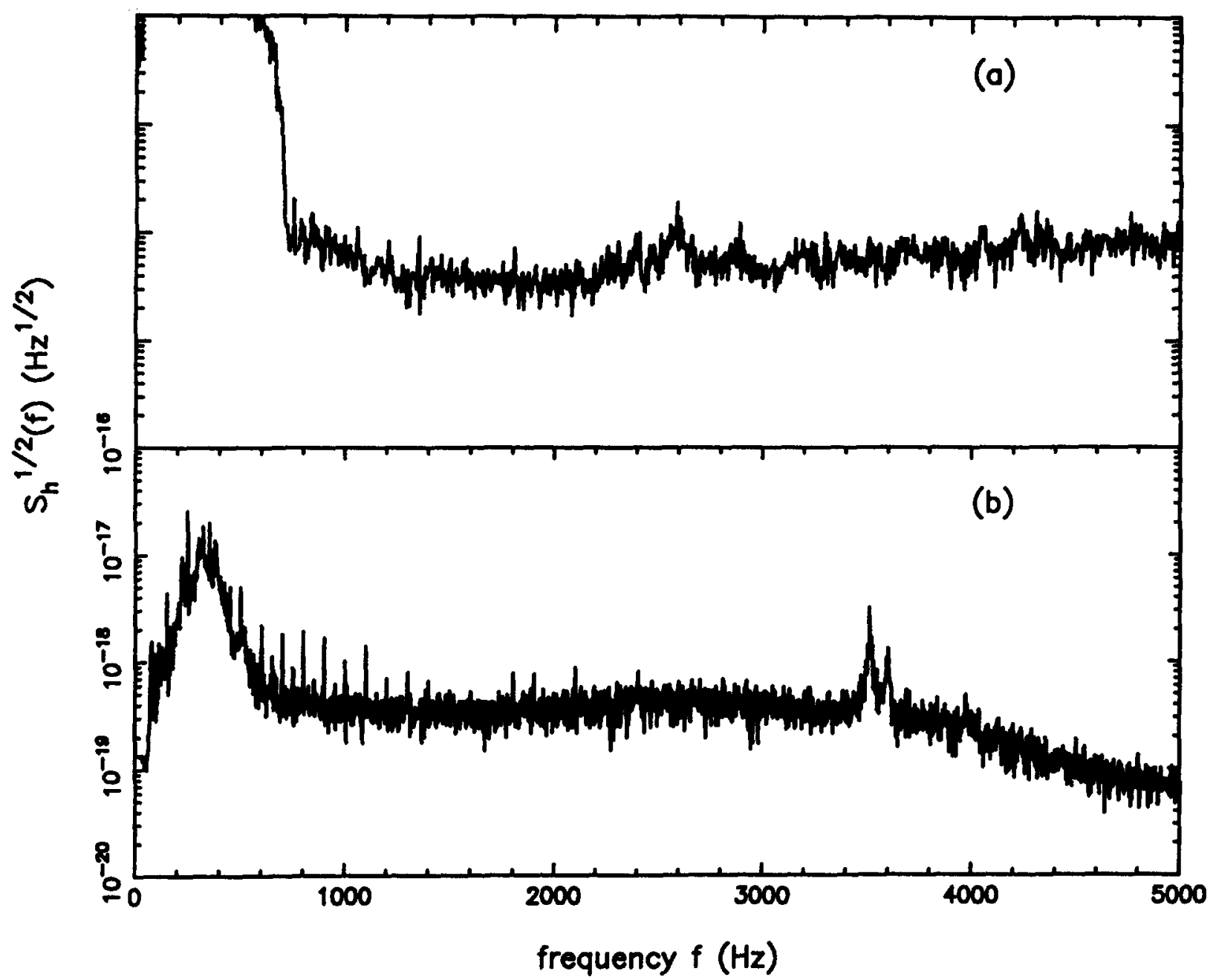

Figure 2: The rms strain noise in the (a) Glasgow and (b) Garching detectors during typical good periods of the observing run. 
the detector. The vetoes were partially but not entirely successful in eliminating periods when the detectors were performing below par. The normal operating sensitivity of Glasgow was $1.5 \times$ $10^{-17}$ and that of Garching was $1.4 \times 10^{-17}$, although there were un-vetoed times when the detectors were behaving five times worse.

The second level accepted only data from periods where the sensitivity of the two detectors was nearly optimum. At other times, the information from the experiment was less useful. Coincidences were only accepted when the detectors' sensitivity was better than $4 \times 10^{-17}$ in a period of $1.6 \mathrm{~s}$. This reduced the total coincidence coverage to about 62 hours.

At the third level, events that survived the first two vetoes were tested against a simple model: if they were genuine gravitational waves, they ought to have shown the same intrinsic strain amplitude in both detectors, apart from the effects of detector noise (recall that the detectors respond almost identically to incident waves). We calculated the probability that the two observed strain amplitudes could have been produced by an unknown signal plus independent noise, given the noise level in each detector at the time of the coincidence, and based on a model of independent Gaussian noise in the two detectors. If this probability was less than $0.1 \%$, we rejected the coincidence. The reason for rejecting such events is our assumption that any real gravitational wave events of this strength will be very rare, and it is therefore unreasonable to conclude that a coincidence is caused by a gravitational wave if to do so requires us to assume that, in addition, the detectors were behaving in a very unusual way as well when that rare event occurred. We call this the h-veto.

Coincidences that survive the three levels of rejection set the upper limit on the sensitivity of our experiment.

\subsection{Results}

We chose a threshold of 4 times the noise for generating events from kiloHertz filters. The lefthand panel of Figure 4 shows the distribution of coincident events that pass the housekeeping vetoes during good data periods (levels 1 and 2). A calculation based on the empirical signal-tonoise distributions of all the events in each detec- tor that survive these vetoes, yields a probability for the least likely coincident event in this diagram of 0.57 , so none of the events is statistically unusual.

The right-hand panel of the same figure shows the events that pass the $h$-veto, so that they could have been generated by a gravitational wave with a reasonable amount of noise on top. The axes here are in strain rather than signal-to-noise. The inferred intrinsic amplitude is the average value of the strains on the two axes. From this figure we conclude that our limit on gravitational waves in our broad passband is $1.6 \times 10^{-16}$. This is the limit if the waves arrive from the zenith with the optimum polarization. 'The rms limit on bursts of any polarization coming from any direction is a factor of $\sqrt{5}$ worse, or $3.6 \times 10^{-16}$.

\subsection{Implications for future experiments}

Our limits are the first obtained over a broad gravitational wave bandwidth. Given the typical rins strain noise of the detectors we estimate that our upper bound on $h$ is only about a factor of 2 worse than the theoretical best limit that these detectors could have set. Recent coincident experiments between resonant bars have set an upper limit of $h=3 \times 10^{-18}\{15]$, but this is for a narrow waveband. Interferometer prototypes have been improved since 1989, and would probably approach this limit in a similar experiment today. However, of even greater interest than the specific limit is the fact that we have tested interferometric observing, and found performance results that are very encouraging for large-scale interferometers. The efforts being made to make LIGO and VIRGO run optimally are well-justified, since the present results show that the optimum sensitivity of such detectors is well within reach.

\section{COALESCING BINARIES}

One of the most important likely sources of gravitational waves for both the ground-based and space-based interferometers are coalescing binary systems. These are binary systems whose components are close enough for gravitational radiation reaction forces acting on the orbital motion to bring them into coalescence in an astronomically short time. We see three pulsar-containing binaries in our neighbourhood of the Galaxy that 
will coalesce within about $10^{8} \mathrm{yr}$, which suggests that such an event will happen somewhere in our Galaxy at least once every $10^{7} \mathrm{yr}$ or so $[16,17]$. Theoretical studies of binary evolution suggest that the rate may be 100 times higher $[18,19]$.

These rates may seem small, since one does not want to wait even $10^{5} \mathrm{yr}$ for an event like this! But detectors should be able to see these events in very distant galaxies. The reason is that a system at a distance of $100 \mathrm{Mpc}$ (five times further than the Virgo cluster of galaxies) emits waves with an amplitude of only a few times $10^{-23}$, but it emits these waves as a wave train with hundreds or even thousands of cycles in the observable frequency range above $40 \mathrm{~Hz}$. This allows one to do matched filtering on the data stream and improve the signal-to-noise ratio by a factor that is roughly the square root of the number of cycles. This means the signals are as detectable as a burst of amplitude nearly $10^{-21}$, so they should be readily detectable in a large volume of space [20]. The expected event rate is more than one per week.

What makes these sources interesting is the amount of information that one can extract from their waveforms. When observed by a network of 3 interferometric detectors, one can infer from the waves the exact distance to the source. This allows a number of studies, including the determination of the Hubble constant [6] and of the statistics of the masses and spins of neutron stars and black holes.

However, to detect the signals optimally and to extract the desired information from them, the waveform must be predicted accurately over the entire observation time, which can last up to 15 minutes. Any small errors that accumulate can put the predicted waveform out of phase with the real one, and lead to a loss of sensitivity and information. Basically, this means that we have to be able to solve the relativistic 2-body problem accurately.

Although the 2-body problem is elementary in Newtonian mechanics, the nonlinearity of general relativity makes it intractable to exact solution in our case. The only way we have at present for getting accurate answers is the so-called PostNewtonian expansion, in which general relativity is approximated by successively higher-order cor-

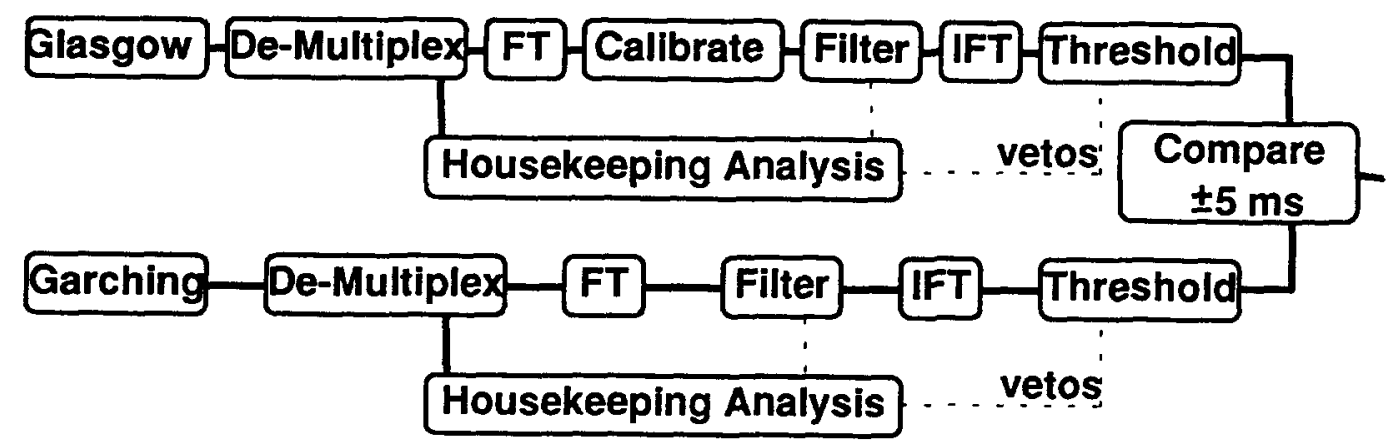

Figure 3: The analysis chain for the search for bursts of gravitational waves. 

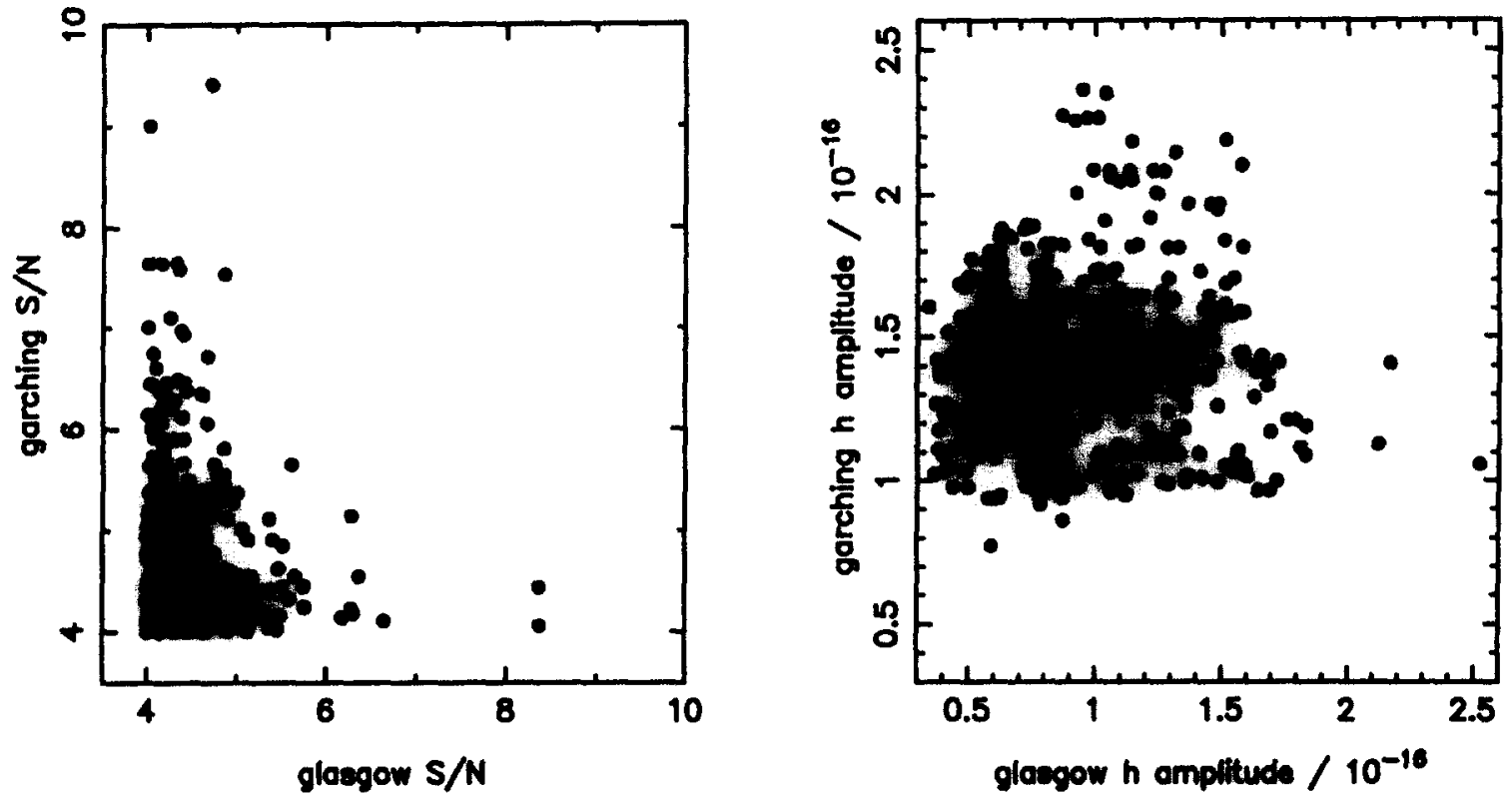

Figure 4: The coincident events passing the first two levels of analysis (left) and then also the $h$-veto (right) for the kilohertz filter. See text for details. 
rections from the Newtonian equations. This can be done consistently at any order, but successive orders rapidly get more complicated.

Cutler et al [21] showed recently that the errors caused by dropping the incompletely-known higher terms in the expansion are significant if observations begin as low as $40 \mathrm{~Hz}$, and moreover it is not clear how many more orders of expansion will be needed to get an accurate predicted waveform.

The situation is thus both tantalising and frustrating. We know in principle that we can extract a great deal of information from these waveforms, but in practice we have not yet developed the adequate theoretical tools. Whether the accurate answer will come from the Post-Newtonian expansions or from other approaches, like full numerical integrations of the two-body problem (an active area of research, but not one that is likely to give good answers quickly), only time will tell. Fortunately, there is some time, since coalescing binaries may not be seen in quantity until LIGO and VIRGO go some way beyond their original sensitivity goals. But the theoretical challenge is formidable, and it is not at all clear that theoreticians will produce accurate waveform predictions before the systems are actually detected!

\section{REFERENCES}

1. B.F. Schutz, in A. Bottino and P. Monacelli (eds.), TAUP 89: Theoretical and Phenomenological Aspects of Underground Physics, Editions Frontieres, Gif-sur-Yvette, 1989 , pp. $167-185$.

2. A. Abramovici et al., Science, 256 (1992) 325 .

3. C. Bradaschia et al., Nucl. Instrum. \& Methods, A 289 (1990) 518.

4. D.G. Blair (ed.), The Detection of Gravitational Waves, Cambridge University Press, Cambridge, 1991.

5. P. Astone, et al, Europhys. Lett., 16 (1991) 231-235.

6. B.F. Schutz, Nature, 323 (1986) 310-311.
7. K. Danzmann, et al, LISA: Proposal for a Laser-Interferometric Gravitational Wave Detector in Space, Max-Planck-Institute for Quantum Optics Preprint 177 (Garching, Germany, 1993).

8. R.W. Hellings, et al, SAGGITTARIUS A Spaceborne Astronomical Gravity-Wave Interferometer, Jet Propulsion Laboratory Engineering Memorandum 314-569 (Pasadena, California, 1993).

9. D. Nicholson, et al, submitted for publication.

10. B.F. Schutz, in Blair, op. cit., pp. 406-452.

11. B.F. Schutz (ed.), Gravitational Wave Data Analysis, Kluwer, Dordrecht, 1989.

12. D.I. Robertson, Ph.D. thesis, University of Glasgow, 1991.

13. T.M. Niebauer, A. Rüdiger, R. Schilling, L. Schnupp, W. Winkler, and K. Danzmann, Phys. Rev. D, 47 (1992) 3106.

14. W.J. Watkins, Ph.D. thesis, University of Wales College of Cardiff, 1991.

15. W.O. Hamilton and H.J. Paik, in R.J. Gleiser, C.N. Kozameh and O.M. Moreschi (eds.), General Relativity and Gravitation 1992, Institute of Physics Publ., London, 1993, p. 407.

16. E.S. Phinney, Astrophys. J., 380 (1991) L17.

17. R. Narayan, T. Piran, and A. Shemi, Astrophys. J., 379 (1991) L17.

18. A.V. Tutukov and L.R. Yungelson, Mon. Not. R. Astron. Soc., 260, (1993) 675-678.

19. H. Yamaoka, T. Shigeyama, and K. Nomoto, Astron. Astrophys., 267 (1993) 433-438.

20. K.S. Thorne, in S.W. Hawking and W. Israel, 300 Years of Gravitation, Cambridge University Press, Cambridge, 1987, pp. 330 458.

21. C. Cutler, et al, Phys. Rev. Lett., 70 (1993) 2984-2987. 